\title{
Highly replicable, low-cost, portable, general-purpose, high-resolution spectrometer with applications in stellar studies and exoplanet science
}

\author{
Clark Baker*a, Guillem Anglada-Escudéa ${ }^{\mathrm{a}}$, Hugh Jones ${ }^{\mathrm{b}}$, William Martin ${ }^{\mathrm{b}}$, \\ ${ }^{a}$ Queen Mary University of London, Mile End Rd, Bethnal Green, London E1 4NS; 'University of \\ Hertfordshire, College Ln, Hatfield AL10 9AB
}

\begin{abstract}
High-resolution spectrometers have proven to be an important tool for astronomical observations and continue to have an ever expanding set of applications, such as high resolution IFS (Integral Field Spectroscopy). With this in mind, we present an alternative approach to the design and construction of Echelle type spectrometers. The usual approach is to drive high resolving power through the use of large gratings and long focal length collimators, leading to great production costs in the order of $\$ 1,000,000$. Our compact, proof-of-concept prototype, via the coupling of adaptive optics, achieves comparable performance and resolution; with a theoretical resolving power R>80,000 in the Vis-NIR regime (500nm$1 \mu \mathrm{m})$ at a cost $<\$ 10,000$. This is attained through the use of COTS (Commercially-Off-The-Shelf) and economically designed components. The overall device footprint is compact, measuring the size of a 'shoe-box', approximately $30 \mathrm{~cm} \times 15 \mathrm{~cm}$. The spectrometer prototype is fibre-fed with a single $10 \mu \mathrm{m}$ fibre and follows a double-pass design - applying a custom designed, $108.24 \mathrm{~mm}$ focal length, collimating and re-focussing lens. The system follows an Echelle type design with high resolution achieved through the use of a compact R4 diffraction grating and a prism as the cross-disperser.
\end{abstract}

Keywords: spectrograph, spectroscopy, echelle, high-resolution, exoplanet, low-cost, replicable, integral field

\section{INTRODUCTION}

Optical spectrometers are devices that take an input light source and separate that source into its constituent wavelengths to produce a resultant output spectrum of wavelength against respective intensity. High resolution spectrometers are spectrometers that are able to resolve difference in wavelength to smaller increments than medium and low-resolution spectrometers; these are generally defined in the visible regime as able to resolve differences in wavelength smaller than the order of $10 \mathrm{pm}$.

High resolution spectroscopy has proven to be an important tool in modern astronomy, from galaxy redshift surveys, such as those completed with the DEIMOS instrument, to stellar evolution studies, using for example the NGSIE instrument, and exoplanet discovery/characterisation with the Doppler method [1] [2] [3].

There are numerous ways to design high resolution spectrometers. The design chosen here is the classic Echelle design, first pioneered by Nagaoka and Mishima in 1923 [4]. This design uses blazed grating facets to maximise throughput in higher diffraction orders; and applies a prism cross-disperser to separate each of the diffraction orders. This spectrometer design produces a 'ladder-like' spectrum with orders which are spatially separated, see figure 1.

*c.e.baker@qmul.ac.uk; phone +44 (0)207 882 3431; www.qmul.ac.uk/spa/people/research-students/profiles/cebaker.html 


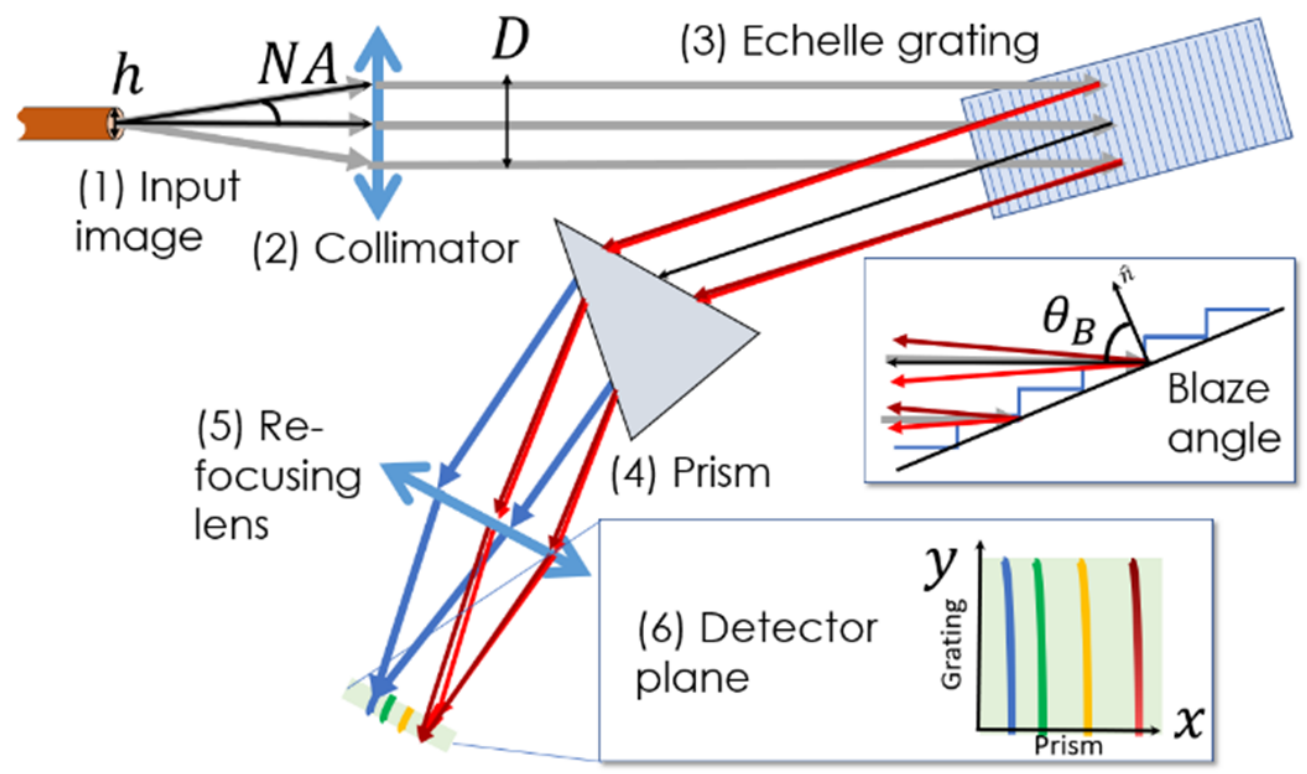

Figure 1. Schematic depicting the layout of an echelle type spectrometer. Light enters through a fibre or slit (1), is collimated by the collimating lens (2), dispersed in high resolution out of the plane of the page by the Echelle grating (3), cross-dispersed parallel to the plane of the page by the prism, spatially separating the orders (4), whereby the light is then refocussed onto a detector by the refocussing lens (5). Item (6) depicts the resultant Echelle spectrum.

With large scale contemporary instrumentation projects hindered by set-backs and ever-increasing budget requirements, such as the production of the JWST, the question is raised as to whether projects of this size, and larger, are feasible going forward. As such, we suggest that the design of instrumentation should be approached in a different way [5]. With this mind-set, we aim to demonstrate that a compact high-resolution spectrometer with applications in Astronomy can be produced in a replicable manner at a low cost.

\section{DESIGN OVERVIEW}

\subsection{Basic considerations for the Echelle design}

Spectrometers are a highly constrained system. Spectrograph design can be seen fundamentally as the trade-off between four main parameters: Resolving Power, Collimator Focal Length, Diffraction Grating Blaze Angle and the Size of the Input; either fibre size or slit width. This is well demonstrated through the Theoretical Resolving Power Equation, equation 1. We provide the details for the derivation here, as understanding this relation is crucial to the design choices discussed later.

If we consider the spectrometer in the high resolution axis only, the prism can be ignored, as shown in figure 2 . In this simplified view, the system can be seen as a collimating lens (lens 1), a grating and a re-focussing lens (lens 2). For a reimaging system consisting of the two lenses only, an object such as the fibre of height $h$, when collimated through lens 1 will produce a beam with angle $\theta$ such that $\tan (\theta)=h / f_{1}$. Where $f_{1}$ is the focal length of lens 1 . Similarly, when this beam is re-imaged through lens 2 the image of the fibre head has height $h_{2}=f_{2} \cdot \tan (\theta)$. Now for 2 wavelengths, $\lambda$ and $\lambda+\delta \lambda$, separated by $\delta \lambda$, the diffraction grating will introduce a difference in beam angle of $\varepsilon$, see figure 2 . When these beams are re-focussed, they will have a separation of $h_{2}^{\prime}=f_{2} \cdot \tan (\varepsilon)$. As a simplification, ignoring diffractive and aberration effects, we state that in order to distinguish a difference in wavelength of $\delta \lambda, h_{2}^{\prime}$ must be greater than or equal to $h_{2}$, hence $f_{2} \cdot \tan (\varepsilon) \geq f_{2} \cdot \tan (\theta)$. Simplifying this gives a critical value when $\tan (\varepsilon)=h / f_{1}$. Taking the grating equation, $m \lambda=n d(\sin (\alpha)+\sin (\beta))$, where $m$ is the diffraction order, $d$ is the groove density (often quoted in 
Grooves $/ \mathrm{mm}$ ), $\alpha$ and $\beta$ are the incoming and outgoing angles at the grating, respectively, and $n$ is the refractive index of the medium that the system is in; which for this example we shall take as vacuum, such that $n=1$. For some wavelength, $\lambda_{L}$, we shall take the Littrow condition to be satisfied, giving $\alpha=\beta=\varphi$; where $\varphi$ is the blaze angle of the grating. This simplifies the grating equation to $m \lambda_{L}=2 d \sin (\alpha)$. For a wavelength separated from $\lambda_{L}$ by $\delta \lambda$, the outgoing angle from the grating will have an additional angle $\varepsilon$, such that $m\left(\lambda_{L}+\delta \lambda\right)=d(\sin (\alpha)+\sin (\alpha+\varepsilon))$. Subtracting and re-arranging these two equations result in $\delta \lambda=\varepsilon d \cos (\alpha) / m$. Taking a small angle approximation for $\varepsilon$, we see that $h / f_{1}=\varepsilon$, therefore $\delta \lambda=h d \cos (\alpha) / m f_{1}$. The definition of resolving power is given by $R=\lambda / \delta \lambda$, of which, through substituting into from the simplified grating equation and our value of $\delta \lambda$, we see that

$$
R=\frac{2 f_{1} \tan \alpha}{h}
$$

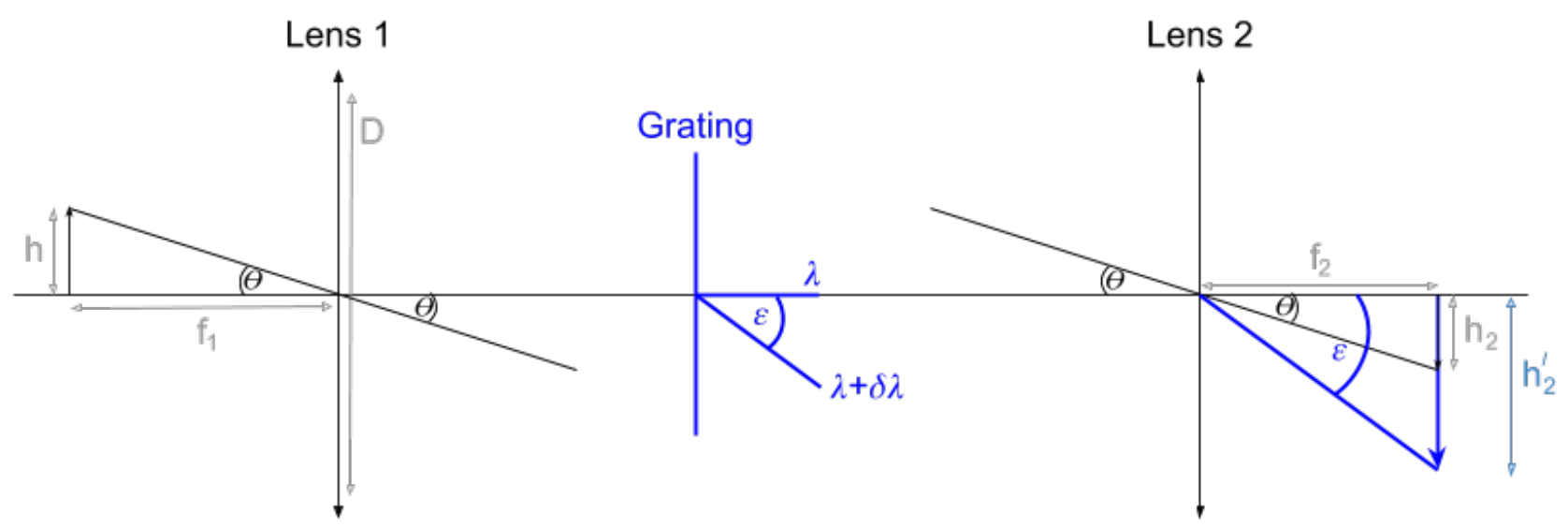

Figure 2. Diagram depicting a simplified spectrometer system, considering the high-resolution dispersion axis only. This figure demonstrates the key parameters used in the derivation of the Theoretical Resolving Power Equation, equation 1.

It is to be noted here that resolving power is not explicitly dependent on grating facet number, which only regulates the wavelength span of the individual diffraction orders, or focal length of the refocussing lens, which will only alter magnification of the final image.

Furthermore, it is possible to relate the telescope diameter to the resolving power of the overall telescope and spectrometer system. In order to maximise throughput, the size of the input fibre, $h$, must match the physical size of a point source imaged to the focal plane of the telescope. For a telescope of focal length, $f_{t}$, the angular image size, $\omega$, is related to the physical image size by $\omega=h / f_{t}$, where it is assumed that $\omega$ is a small angle; for a star, this is of the order of 1 arcsec. Following the definition of the F-number, the telescope's F-number is described by $F_{t}=f_{t} / D_{t}$, where $D_{t}$ is the diameter of the telescope. With the aim to conserve etendue, the F-number of the spectrometer, $F_{s}$, and the telescope must be matched; such that $F_{t}=F_{s}=F$. Following this, it can be seen that $h=F D_{t} \omega$ and $f_{s}=F D_{s}$; furthermore, substituting these expressions into equation 1 , we see that

$$
R=2 \frac{D_{s}}{D_{t}} \frac{\tan \alpha}{\omega}
$$

The spectrometer has been designed to be coupled to a $0.5 \mathrm{~m}$ telescope with an $F=4.51$ such that its focal length is $2.255 \mathrm{~m}$. By forcing the telescope beam to fit the numerical aperture of commercially available optical fibres (NA $\sim 0.11$ ), the physical image of a point star becomes $10 \mu \mathrm{m}$ for a seeing of 1 arcsecond. The diffraction limited image of such a telescope would have a diameter of approximately $4 \mu \mathrm{m}$, meaning that our input is 2.5 times larger than it would strictly 
need to be for a diffraction limited instrument. It can be shown that this relation holds for telescopes or arbitrary sizes. As long as a moderate performance AO (Adaptive Optics) system is operational (which can correct the image to smaller than 2.5 times the diffraction limit of the instrument), our spectrometer can be efficiently coupled to any arbitrarily large aperture.

Hence, with AO, a small inexpensive spectrometer could be effectively coupled to a telescope as large as the EELT or, more productively, an array of high-resolution spectrometers could be fed by an integral field fibre array, opening a widearray of observational possibilities. This offers a scalable way (linear with the number of units) to equip large telescopes with high resolution spectrometers; and enables new exciting science cases [6].

\subsection{Top level requirements}

The spectrometer aims to be applicable to atomic and molecular spectroscopy. Given the typical conditions in main sequence stars, this can be achieved with a resolving power of $R>50,000$. Furthermore, $R \approx 100,000$ typically provides a 'sweet-spot' between high spectral resolution and light collection efficiency [7]. The optical to NIR bands are required in many cases for exoplanet science [7]. As such, our spectrometer is designed to operate between 500nm and 1um with the overall aim of achieving as broad a wavelength range as possible in a single device. The device must be low-cost, with the prototype having a total component value less than $\$ 15,000$ and with a finalised replicable unit manufacturable for under $\$ 10,000$. A summary of these and other requirements are given in Table 1.

Table 1. Summary table of the instrument requirements.

\begin{tabular}{|c|c|c|}
\hline Property & Technical Requirement & Goal \\
\hline $\begin{array}{l}\text { Spectral } \\
\text { Resolution }\end{array}$ & $\begin{array}{l}>50,000 \text {, sufficient to resolve atomic and molecular spectral lines on main } \\
\text { sequence stars and planets. This resolution is also sufficient to resolve the } \\
\text { signature of Earth atmospheric molecular gases. Satisfying this condition } \\
\text { requires both careful selection of the spectrometer's dispersive elements and } \\
\text { stringent constraint on the system's image quality. }\end{array}$ & $\begin{array}{l}100,000 \text { achievable in a slightly reduced } \\
\text { throughput mode by reducing the size of the } \\
\text { input image. Such a mode could be very useful } \\
\text { in high-S/N applications (solar and air gas } \\
\text { analysis). }\end{array}$ \\
\hline $\begin{array}{l}\text { Wavelength } \\
\text { Coverage }\end{array}$ & $\begin{array}{l}\text { 600-900nm. This is the wavelength range where: adaptive optics systems } \\
\text { perform well (in the optical), nearby sun-like stars down to red-dwarfs have most } \\
\text { of their flux, and numerous Earth atmosphere gases have strong spectral } \\
\text { signatures (eg. } \mathrm{O}_{2}, \mathrm{H}_{2} \mathrm{O} \text { ). This wavelength range also cover wavelengths where } \\
\text { toxic gases such as } \mathrm{HCl}, \mathrm{HF} \text { have numerous absorption features. }\end{array}$ & $\begin{array}{l}500-1000 \mathrm{~nm} \text {. On the blue end, it would add } \\
\text { substantially more information on Sun-like } \\
\text { stars, and on the red end it would enable } \\
\text { monitoring of additional gases with strong } \\
\text { absorption bands at } \sim 1 \text { micron (eg. } \mathrm{CH}_{4}, \mathrm{C}_{2} \mathrm{H}_{2} \text {, } \\
\mathrm{NH}_{3} \text {, etc.) }\end{array}$ \\
\hline $\begin{array}{l}\text { Wavelength } \\
\text { Calibration } \\
\text { \& Stability }\end{array}$ & $\begin{array}{l}\text { Able to provide } \sim 5 \mathrm{~m} / \mathrm{s} \text { precision in the measurement of Doppler shifts of stellar } \\
\text { or telluric atmospheric lines during integrations of } \sim 600 \mathrm{sec} \text {. This mainly } \\
\text { imposes constraints on mechanical and temperature stability. }\end{array}$ & $\begin{array}{l}<2 \mathrm{~m} / \mathrm{s} \text { precision for } 60 \mathrm{sec} \text { integration on Solar } \\
\text { observations and bright stars. }\end{array}$ \\
\hline $\begin{array}{l}\text { Efficiency } \\
\text { (from input } \\
\text { to detector) }\end{array}$ & $\begin{array}{l}>30 \% \text { for } 600-900 \mathrm{~nm} \text { to remain competitive with existing echelle spectrometers. } \\
\text { Moderately high efficiency is also needed to keep integration times low in high- } \\
\text { contrast imaging applications. }\end{array}$ & $\begin{array}{l}>60 \% \text { for } 500-1000 \mathrm{~nm} \text {, at least on specific } \\
\text { ranges where Quantum Efficiency }(\mathrm{QE}) \text { of the } \\
\text { detectors is higher. }\end{array}$ \\
\hline $\begin{array}{l}\text { Abs. Wave- } \\
\text { length } \\
\text { Calib. }\end{array}$ & $<2 \mathrm{~m} / \mathrm{s}$ precision per calibration set (before and after each observation). & $<2 \mathrm{~m} / \mathrm{s}$ precision (once per night). \\
\hline Size & $360 \mathrm{mmX} 260 \mathrm{mmX} 120 \mathrm{~mm}$, the size of a conventional shoe-box. & $\begin{array}{l}300 \mathrm{mmX} 100 \mathrm{~mm} \times 100 \mathrm{~mm} \text {, the size of a } 3 \mathrm{U} \\
\text { cube-sat. }\end{array}$ \\
\hline Cost & $\$ 15,000$ & $\$ 10,000$ \\
\hline
\end{tabular}

\subsection{Technical specification and constraints}

With our design approach, minimising instrument cost is a key element. As such we are restricted to the use of COTS diffraction gratings, which mostly consist of replica gratings. While the availability of COTS gratings in recent years is far greater than it has ever been before, the number of available options are still limited. Due to this, the grating was the first component to be chosen; beginning the process of constraining the system design. The Thorlabs GE2550-0875 Echelle 
Grating (79.0 Grooves/mm, 75deg Blaze Angle, $25 \mathrm{~mm}$ x $50 \mathrm{~mm}$ ) was selected, due to its large, almost R4 blaze angle at a very low-cost; along with its availability. Ideally a grating with a lower number of Grooves/mm, such as Thorlabs' 31 Grooves/mm offering, would have been chosen as this would form a spectra with a larger number of shorter orders, leading to a squarer shaped echelle spectrum. This would reduce the amount of anamorphic magnification observed, further discussed in section 3, and also allow the spectrum to better fit onto conventional CCD sensors. Unfortunately, this grating is only offered with an R2 blaze angle, which would not allow for the required resolution to be achieved.

With the diffraction grating chosen, the diffraction grating blaze angle parameter is set to 75deg; and with the diffraction grating width of $25 \mathrm{~mm}$, the size of the aperture stop for the system is set to a diameter of $24 \mathrm{~mm}$. The width of the diffraction grating places a constraint on the collimated beam diameter that the diffraction grating can accept. The beam diameter was chosen to be $24 \mathrm{~mm}$ to avoid using the very edge of the grating where it is expected that performance is degraded through the grating's manufacturing process. Ideally the grating would have a height of $100 \mathrm{~mm}$, such that when it is operated at $\mathrm{R} 4$, the projected aperture is $100 \mathrm{~mm} \cdot \cos (75 \mathrm{deg})=25.88 \mathrm{~mm}$; encompassing the entire beam. Unfortunately, Thorlabs only produce gratings of height $50 \mathrm{~mm}$, giving a projected aperture of $50 \mathrm{~mm} \cdot \cos (75 \mathrm{deg})=12.94 \mathrm{~mm}$, which is less than the $24 \mathrm{~mm}$ beam diameter. Fortunately, due to the geometry of a circular, gaussian beam incident on a rectangular grating cross-section projection, the majority of the light intensity in the beam cross section is accommodated by the grating.

As can be seen in equation 1, another way to increase resolving power is to reduce the size of the optical fibre. It is to be noted that the image size and the numerical aperture are set by the image size and numerical aperture producible by the telescope, for efficient coupling. As discussed in section 2.1, a fibre size of 10um with 0.11 NA allows for the efficient coupling of the spectrometer with a $0.5 \mathrm{~m}$ telescope. The closest COTS component to this is the Thorlabs M65L, which has a $0.1 \mathrm{NA}$. This was used for prototype testing and due to this, the current prototype has some loss in throughput. A 0.11 NA fibre could be cheaply manufactured for the final system to remedy this. This value sets our third constraint on the spectrometer system, as such this now limits our last component of interest: the collimating lens. As the fibre will accept and re-emit light with a numerical aperture of 0.11 , or equivalently an F-number of 4.51 , we can determine the required focal length of the collimating lens, $f_{s}$, from the definition of the F-number and the required beam diameter of 24mm: $f_{s}=F D_{s}=4.51 \times 24 \mathrm{~mm}=108.24 \mathrm{~mm}$. As such, the collimating lens requires a $108.24 \mathrm{~mm}$ focal length to produce the required $24 \mathrm{~mm}$ beam diameter with the $0.11 \mathrm{NA}$ optical fibre. With the key parameters set, the theoretical resolving power of the system can be estimated. Using equation 1 with $f_{1}=108.24 \mathrm{~mm}, \alpha=75 \mathrm{deg}$ and $h=10 \mathrm{um}$, it is found that $R=81,000$.

Through the use of such a compact grating, with the resulting small collimated beam size and the short collimating lens focal length that comes from this, an instrument can be constructed with a high resolving power and a highly compact size; at around the size of a shoe-box.

Table 2. Summary table of key design parameters once constrained to a feasible combination of COTS components.

\begin{tabular}{|c|c|c|c|c|c|c|}
\hline Fibre size & Fibre NA & $\begin{array}{l}\text { System } \\
\text { F/\# }\end{array}$ & $\begin{array}{l}\text { Collimating Lens } \\
\text { Focal Length }\end{array}$ & Blaze Angle & $\begin{array}{l}\text { Facets } \\
\text { (Grooves/mm) }\end{array}$ & $\begin{array}{l}\text { Theoretical Resolving } \\
\text { Power }\end{array}$ \\
\hline 10um & 0.11 & 4.51 & $108.24 \mathrm{~mm}$ & 75deg & 71 & 81,000 \\
\hline
\end{tabular}

\section{OPTICAL AND MECHANICAL DESIGN}

As discussed in section 2.1, the focal length of the re-focussing lens is a free parameter in terms of resolving power performance. The ratio of the collimating lens and re-focussing lens' focal lengths determine the magnification of the 
spectra image in the focal plane. Given this, the main aim of the re-focussing lens' design is to magnify the spectra image to allow optimal Nyquist sampling on the chosen detector.

With this in mind and the aim to keep the system as compact as possible, the approach taken to the optical layout of the spectrometer was given substantial scrutiny. There are two ways the optical layout can be designed:

- $\quad$ Single pass, where the system has a separate collimating and re-focussing lens, shown in figure 1 and as seen in the design of UVES or HARPS [8][9].

- Double pass, where the system uses one lens for both the collimating and re-focussing of the beam, as seen in PFS [10].

Both of these optical layouts have their intrinsic pros and cons. For this system, the double pass design was chosen to maximise compactness and to minimise the number of components; reducing overall system cost. In addition to this, the beam reflection angle at the grating (in the cross-dispersion axis) can be substantially reduced for a compact double pass system, leading to a more symmetric system, hence reducing anamorphic magnification effects [11].

\subsection{The aperture stop}

A very important item of note in the design of spectrometer systems is the location of the aperture stop. This can be defined as the point where all of the principle rays of the system cross each other at the optical axis [12]. In a spectrometer system the location of this aperture stop can be seen to be at the location of the diffraction grating, as that is where all of the principle rays cross the optical axis during dispersion. In the majority of commercial lens system, such as camera lenses, the aperture stop is located within the lens itself. As such, a custom optic must be designed for the collimating/refocussing lens.

\subsection{Optical design parameters and requirements}

The collimating/refocussing lens, henceforth known as the lens, was designed to have the aperture stop $135 \mathrm{~mm}$ away from the front element of the lens. This was chosen as a good estimate to the placement of the diffraction grating, accommodating the physical placement of both the prism and the grating. This aperture stop placement allows the lens to accommodate beams traveling away from the optical axis when they reach the lens' front element, focussing them back towards the image plane. See figure 3 which demonstrates the lens' function and how this differs from a standard camera lens.

With the aperture stop placement set, the next most important set of requirements for the lens design are field angle acceptance and required wavelength range. The latter of which was chosen to be 500nm-1um with the aim to achieve diffraction limited performance across this entire range. In order to determine the field angle acceptance required by the lens, the dispersion angles produced by the diffraction grating and the prism must be characterised.

For the diffraction grating, the maximum dispersion angle of interest is determined by the first minima of the blaze function. The intensity of the blaze function is dictated by the blaze equation [13],

$$
I=\frac{\sin \left(\frac{m \pi \cos (\varphi) \cdot[\sin \tilde{\alpha}+\sin \tilde{\beta}]}{2 \tan \varphi \cos \tilde{\alpha}}\right)^{2}}{\left(\frac{m \pi \cos (\varphi) \cdot[\sin \tilde{\alpha}+\sin \tilde{\beta}]}{2 \tan \varphi \cos \tilde{\alpha}}\right)^{2}}
$$



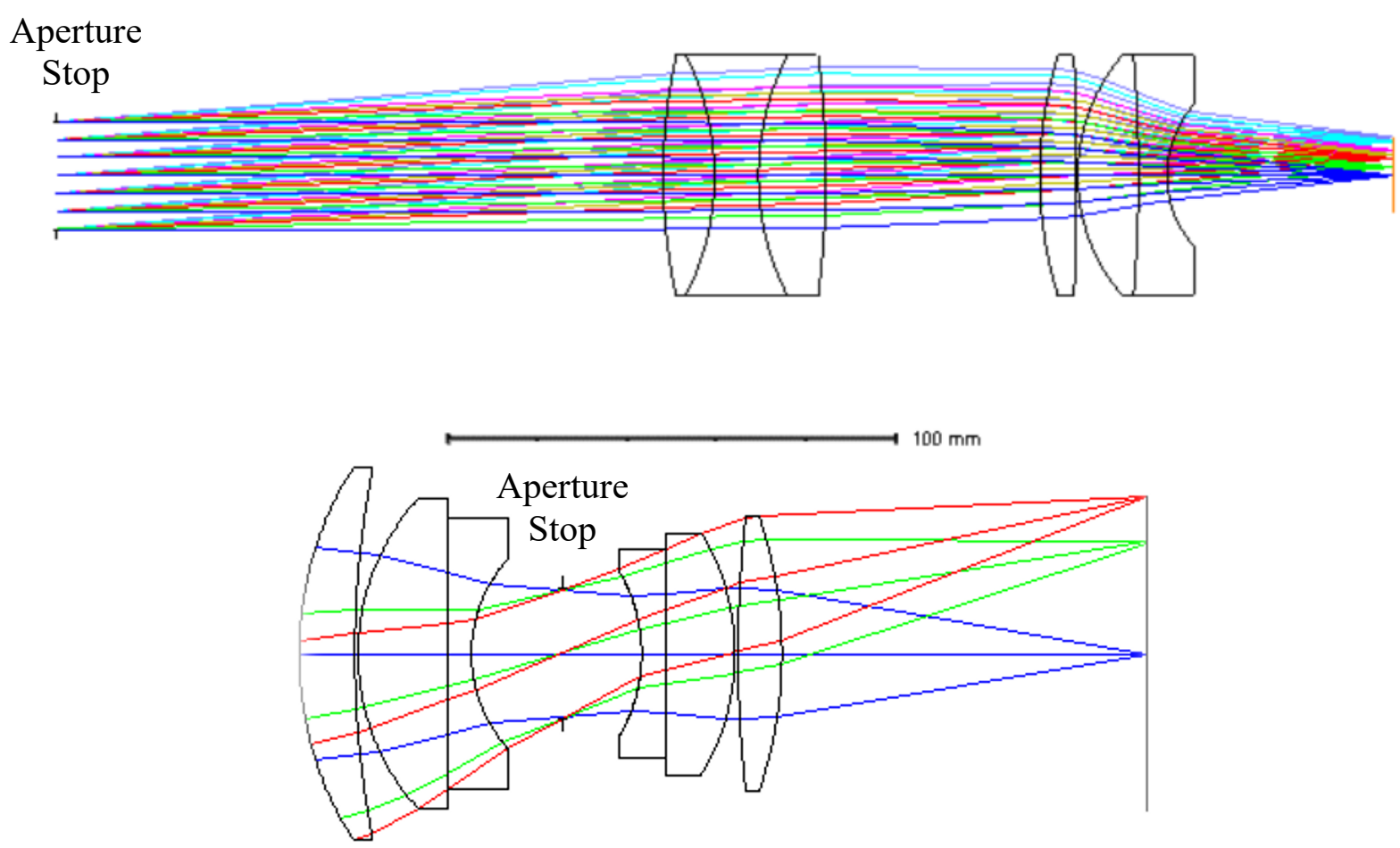

Figure 3. Schematic depicting the location of the aperture stop for a spectrometer's collimating/re-focussing lens (top panel) and a conventional lens, a Double Gauss design in this case (bottom panel).

where $m$ is the dispersion order number, $\varphi$ is the blaze angle and $\tilde{\alpha}=\alpha-\varphi$ and $\tilde{\beta}=\beta-\varphi$ are the input and output angles relative to the grating facet normal. Working on Littrow, $\alpha=\varphi$ and hence $\tilde{\alpha}=0$. Using this and simplifying equation 3 , it is found that

$$
I=\frac{\sin \left(\frac{m \pi \sin \tilde{\beta}}{2 \sin \varphi}\right)^{2}}{\left(\frac{m \pi \sin \tilde{\beta}}{2 \sin \varphi}\right)^{2}}
$$

Using the grating equation for a grating with 79.0 Grooves $/ \mathrm{mm}$ and a $75 \mathrm{deg}$ blaze angle, a test wavelength of $800 \mathrm{~nm}$ is found to be in order 30 . Substituting these values into equation $2 \mathrm{~b}$, the first minima of the blaze intensity function is found at $3.692 \mathrm{deg}$. As such, the lens should be designed to accept at least a field angle from the optical axis of $3.692 \mathrm{deg}$. Note that the blaze function is widest at the lowest order, so the lower orders down to order 24 at a wavelength of $1 \mu \mathrm{m}$ should be expected to be slightly wider than this.

The dispersion of the prism can be characterised at the angle of minimum deviation by [14]

$$
\delta=2 \sin ^{-1}\left(n \cdot \sin \left(\frac{\gamma}{2}\right)\right)-\gamma
$$


where $\delta$ is the deviation angle, $n$ is the refractive index of the prism and $\gamma$ is the angle of the prism wedge.

For an N-BK7 prism with a dispersion angle of $\gamma=45 \mathrm{deg}$, the total deviation in field angle between the lowest and highest order is found using equation 5. The refractive index for our extreme wavelengths are as follows, $n_{500 \mathrm{~nm}}=1.5214$ and $n_{1000 \mathrm{~nm}}=1.5075$. Hence, substituting into equation 4 we find $\delta_{500 \mathrm{~nm}}=26.21 \mathrm{deg}$ and $\delta_{1000 \mathrm{~nm}}=26.47 \mathrm{deg}$, leaving the difference in dispersion angle of $\Delta \delta=\delta_{1000 \mathrm{~nm}}-\delta_{500 \mathrm{~nm}}=0.745 \mathrm{deg}$. Therefore, the field angle introduced by the prism is $0.745 \mathrm{deg}$. Further to this, it is found that this prism gives a separation of 5.215 fibre widths between orders, allowing for a compact spectrum as required.

Given this, the total deviation in field angle introduced by a $45 \mathrm{deg}$ N-BK7 prism is found to be $0.745 \mathrm{deg}$. Following these two field acceptance requirements, the lens should be designed to accept a minimum field from the optical axis of 3.692deg. It was decided to set the minimum field acceptance angle from the optical axis to be $5 \mathrm{deg}$, giving $10 \mathrm{deg}$ of field acceptance in both directions. This allows for the introduction of some additional field angle from the beam reflection angle at the grating (in the cross-dispersion axis) seen in the double pass system, as well as giving opportunity later in the design process to alter the optical layout or use a grating with a wider blaze function.

\subsection{Imaging performance target}

A point source object will be imaged through the spectrometer to a finite size due to the diffraction limit and optical aberrations. The fibre head has finite size and so it can be thought of as made up of an array of 'many' point sources. The image produced by the fibre is hence a convolution of the fibre's perfect image and the point source image. Hence, the size of the final image, $U$, is the sum in quadrature of the fibre size, $h$, and point source spot size, $p$, such that

$$
U=\sqrt{(h)^{2}+(p)^{2}} .
$$

Given the fibre head size is $10 \mu \mathrm{m}$, the spot diameter of the lens' re-imaging of a perfect point source can be allowed to reach approximately $6 \mu \mathrm{m}$ with minimal increase in image size and hence loss in resolution.

\subsection{Detailed lens design and manufacturability}

Throughout the lens design process, the aim was to minimise the number of optical surfaces in order to maximise throughput. Initial testing in both in the laboratory and using Zemax models found, as expected, that singlet and doublet lenses do not have the necessary degrees of freedom to achieve the near diffraction limited performance across the field and wavelength range required. As theoretically understood, a triplet lens is the lowest number of elements required to provide the necessary number of degrees of freedom to correct for all of the Seidel aberrations [15]. Whilst, ideally, a triplet would be applicable as our double pass lens system, we aim to produce the spectrometer at a low-cost. Hence, our glass selection is greatly reduced to that which is readily available and low in cost. With this limitation in glass selection, and hence limitation in refractive index and Abbe number of the lens elements, it was found that the minimum number of elements required to achieve near diffraction limited performance following our wavelength and field criteria was 6 elements.

The initial lens design was based on the well-studied Double Gauss lens with the aperture stop placed at the required $135 \mathrm{~mm}$ away from the front element of the lens. The Double Gauss was selected due to its high resolution, diffraction limited imaging performance at large field angles across a wide wavelength range. It was found, as expected, that applying such a significant stop shift to the lens, its properties varied significantly from its initial design. With this began the optimisation and re-design process. 
Through the use of Zemax optical studio, the lens was re-optimised and redesigned until its imaging performance met the 10um spot diameter criteria or, if it came first, diffraction limited performance for the field and wavelength requirements specified. It is to be noted that at this stage of the design process, the lens was optimised purely as a re-focussing lens. The design was approached in this way as when the lens was placed into the full spectrometer model, due to the double pass set-up, the number of degrees of freedom for Zemax to optimise were too great to lead to applicable results in a reasonable timescale. Once a design that fit the design criteria was found, the manufacturability/procurement process was begun.

Prior to contacting manufacturers, the lens tolerances were tested using Zemax's tolerancing tools. This comprised of setting tolerances for all of the lens parameter and running a series of, say 3000, Monte-Carlo simulations to produce systems within the parameter space set by the tolerances and observing the performance of these systems. If less than $95 \%$ of the systems performed as expected, then the tolerances were tightened where necessary, with the Zemax tolerancing tools highlighting which tolerance had the largest effect on performance. This was repeated until the performance of $95 \%$ of the simulated systems performed as required.

The procurement stage of the optical design was unexpectedly the longest single stage of the entire instrument design. Between an applicable design being created in Zemax and a manufacturable, low-cost, procurable design being reached was approximately 9 months of design work. The design went through 8-10 major redesigns with input from manufacturers. The process would commence as follows: a manufacturer would be approached and they would give us a list of criteria to meet in order to bring the lens to a manufacturable state. In terms of design, these criteria would generally relate to glass selection, lens thickness, edge thickness, surface curvature or overall system tolerances. In addition to this, each of the lens parameters would require reasonably loose tolerances in order to be manufactured at a low-cost. Once a design was iterated with the manufacturer a number of times until it met these criteria and also still fulfilled our performance requirements, we would return to the manufacturer for a quote. Unfortunately, we found on multiple occasions that manufacturers would either no longer be interested in producing a small batch of lenses, the quote would beyond the scope of this project or the tolerances required would be too tight. This then began a cycle until we found Kingsview Optical [16]. We can highly recommend them, they worked closely with us during the manufacturing iteration process, giving clear instruction throughout.

Unfortunately, the period of which this lens was designed was prior to Zemax's optimisation system including a merit function operand; to allow for robust optimisation to maximise manufacturability by finding designs with loose tolerances that achieve close to nominal performance. As such, whilst designing, the required tolerances of a design were effectively beyond our control. We had to accept the tolerances that the design required, which made the procurement process far more difficult. Note, the TOLR operand existed but this was found to be minimally effectual with our design. Zemax's new 'design for manufacturability' option greatly improves the ease of design and the procurement process.

During the design stage, it was possible to significantly improve spot size performance across the field and wavelength range by ignoring lateral chromatic aberration during the optimisation process. This was possible due to the nature of the spectrometer system. As each of the images formed in the image plane will be monochromatic re-images of the fibre head at every wavelength down to the resolution of the spectrometer. Given that each of the images will be monochromatic, we do not need to be concerned with lateral chromatic shift effecting resolution, it will only change the location of the image. The location will also change minimally over the course of an individual order as the wavelengths are all sufficiently close together.

With the final iteration of the manufacturable lens, the design was refined to have only 9 surfaces with 6 elements; as 4 of the surfaces were cemented while maintaining the required optical performance, see figure 4 . Each of the elements were set to 2 " in diameter in order to maximise ease of manufacturability and ease of mounting; allowing all of the elements to be placed in a 2 "tube with toleranced spacers to ensure the required element separation. 
Another approach used to reduce manufacturing cost on this prototype was to apply only a single layer MgF2 anti-reflective coating on the outer element surfaces. Further production, now that the optical performance has been proven to be sufficient, will be produced with higher performance anti-reflective coatings on all surfaces, increasing the cost per lens in the order of $\$ 500$ but greatly increasing throughput.

It was found that the key tolerances for the final design were the lens curvatures, specifically the low radii elements which had to be set to within the order of $10 \mu \mathrm{m}$ from the nominal value. Element thickness, tilt and decentre were all able to be set to standard uniform values of $5 \mu \mathrm{m}, 0.01 \mathrm{deg}$ and $4 \mu \mathrm{m}$, respectively. The Abbe number tolerance was set to $0.2 \%$ and the refractive index was set to $\pm 5 \times 10^{-4}$, both following industry standards [17].

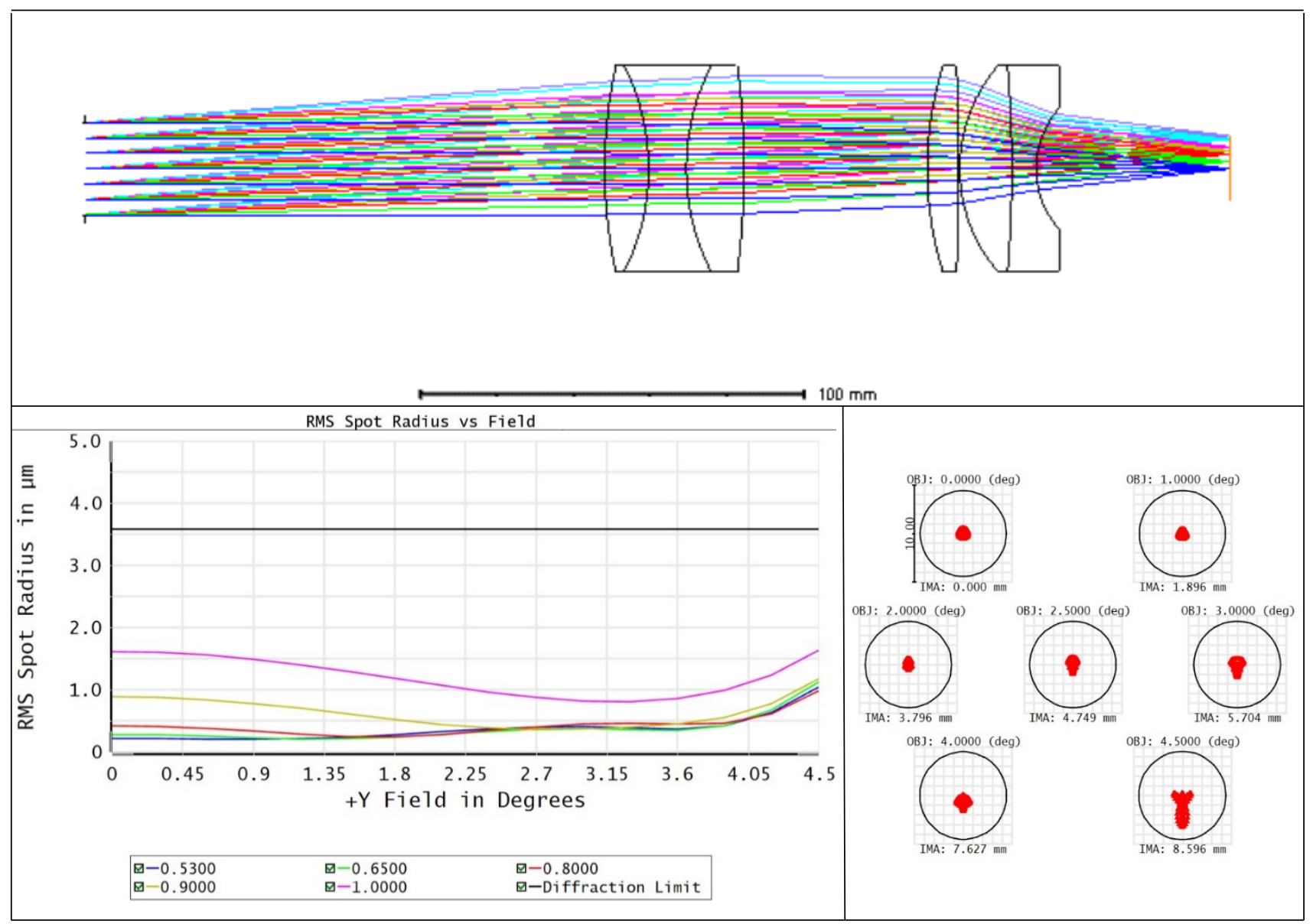

Figure 4. (Top panel) Schematic depicting the geometry of the finalised manufacturable lens design. (Bottom-right panel) Spot diagram for the lens design, with a sub-plot for each of the chosen field angle values from $0 \mathrm{deg}$ to $4.5 \mathrm{deg}$. The red spots depict the aberration limited spot size and the black circles depict the diffraction limit for the system. (Bottom-left panel) Graph of field angle against RMS spot radius for the lens system. The black line represents the diffraction limit value, the blue line represents $530 \mathrm{~nm}$, the green line represents $650 \mathrm{~nm}$, the red line represents $800 \mathrm{~nm}$, the yellow line represents $900 \mathrm{~nm}$ and the pink line represents $1000 \mathrm{~nm}$. It can be seen that the lens is diffraction limited across this field and wavelength range.

In order to test the lens' performance, after each iteration it was placed into a model spectrometer in Zemax using the double pass layout, see figure 5 (top panel). The performance of the resultant spectrometer system was measured through the spot size of a number of wavelengths outlining the centre and each of the extreme wavelengths in the diffraction orders. 
For the nominal final lens design and spectrometer layout, it can be seen that diffraction limited performance is achieved across the entire spectrum, see figure 5 (bottom panel). The worst performing version of the lens found in 3,000 of Zemax's tolerance Monte-Carlo simulations, was placed into the spectrometer system. This system was found to have close to diffraction limited performance across the entire spectra. Given this, we were then sufficiently happy with the lens design in order to move into procurement and have the system manufactured.

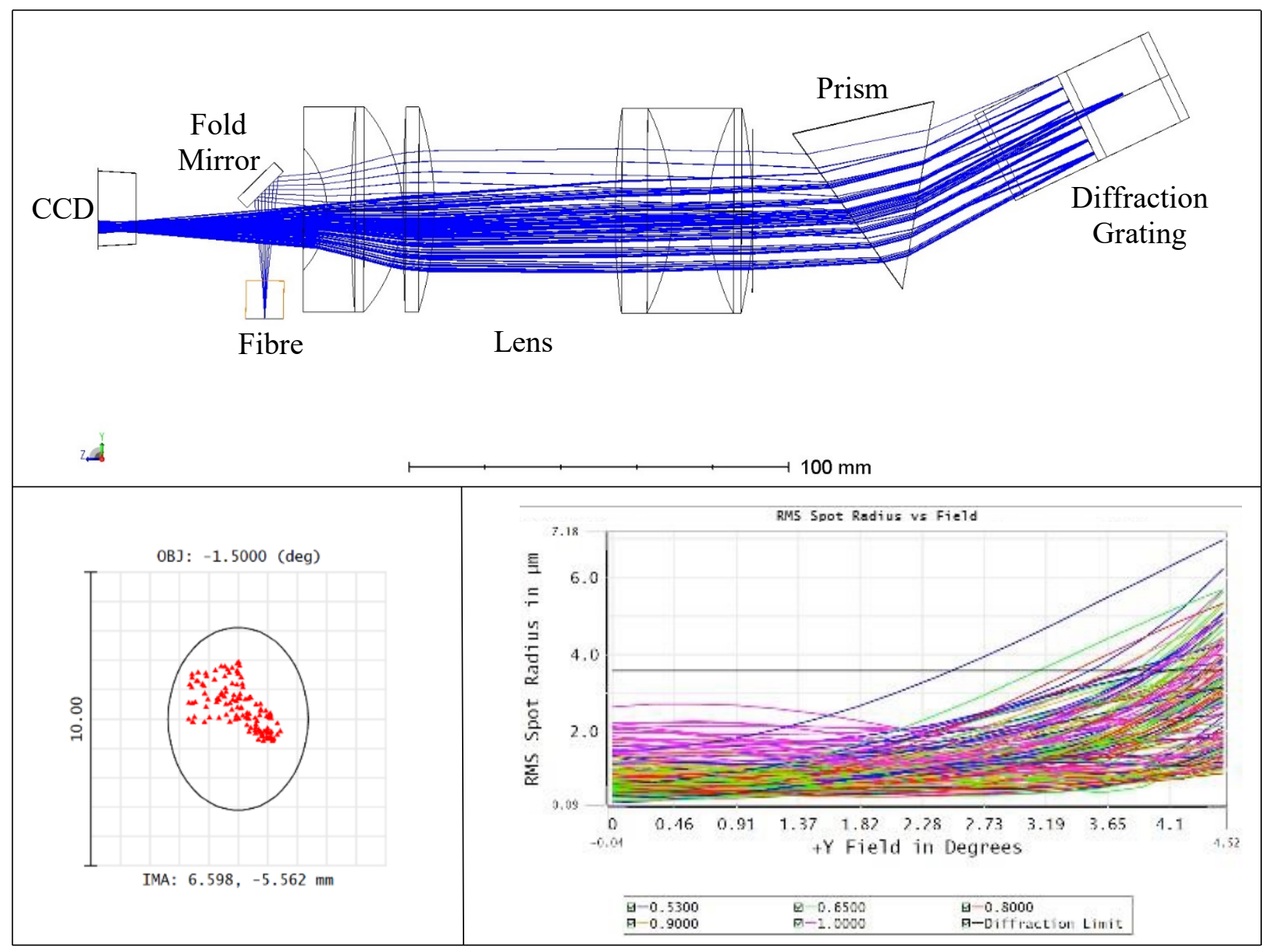

Figure 5. (Top Panel) Schematic layout of the finalised double-pass spectrometer system. (Bottom-left) An example plot of the spot diagram for the spectrometer system at $530 \mathrm{~nm}$, which is at a field of $1.5 \mathrm{deg}$ for this spectrometer system. The red spots depict the aberration limited spot size and the black circles depict the diffraction limit for the spectrometer. The RMS radius of this spot is $1.2 \mu \mathrm{m}$ and the diffraction limit is $2.92 \mu \mathrm{m}$. (Bottom-right) Graph of field angle against RMS spot radius for the manufacturable lens system. This plot contains data for 3000 lens systems that have been generated by Monte-Carlo simulation, taking lens parameter values from within the accepted tolerances. The black line represents the diffraction limit value, the blue line represents $530 \mathrm{~nm}$, the green line represents $650 \mathrm{~nm}$, the red line represents $800 \mathrm{~nm}$, the yellow line represents $900 \mathrm{~nm}$ and the pink line represents $1000 \mathrm{~nm}$. It can be seen that the lens is diffraction limited across most of the field for all of our chosen wavelengths, with acceptable aberration limited performance seen in some of the 3000 systems beyond a field of $3 \mathrm{deg}$.

The lens, with mounting in a tube to the tolerances dictated above and procured in a batch of 2 , was quoted to be manufacturable for $\$ 5500$ per lens. This is the highest cost component of our spectrometer but now that the tooling is produced, with production of higher quantities, the cost per lens has been quoted to drop below $\$ 1500$, making this design replicable at a low cost. 
The detector should be chosen to allow Nyquist-Shannon sampling. Our resultant fibre image with aberration effects is expected, following equation 6 and noting that in the double pass system there is no image magnification, to be approximately $12-14 \mu \mathrm{m}$. As such, when it comes to pixel size, we aim to have at least 2 pixels per fibre head image. Given this, pixels should be smaller than 6-7 $\mu \mathrm{m}$. For our prototype the Starlight Xpress SXVR-H16 was used due to ease of availability. It has a Kodak CCD chip: 2048 x 2048 square pixels in a 15.15 x $15.15 \mathrm{~mm}$ array with each pixel of $7.4 \mu \mathrm{m}$. This sensor is under-sampling the spectrum and the square sensor shape does not fit the rectangular shaped echelle spectrum well. A detector like the Atik 490EX with $3379 \times 2703,3.69 \mu$ m pixels in a 12 x 10 mm array guarantees that the spectrum would be well sampled under Nyquist sampling theorem, with 3.79 pixels per resolution element [18]. Its size is also more than sufficient to cover the image of the echelle spectrum, with its approximately $12 \mathrm{~mm}$ high resolution dispersion from grating and $2 \mathrm{~mm}$ cross dispersion from the prism.

Another option would be a CMOS sensor, which have far better read-out rates and read-out noise performance. Unfortunately, these options are still early in development and as such remain too expensive for current low-cost systems; but this is likely to change in the next few years. We currently use an inexpensive CMOS detector, Thorlabs DCC1645C, for alignment and optical quality measurements, which is made far easier with the fast read out rates. Unfortunately, this detector's sensor is too small and prone to thermal noise to be used for the science data.

\subsection{Lens testing and characterisation}

Once the procured lens was brought into the laboratory, it was characterised to ensure that its performance was as expected from the Zemax model, with tolerances considered. To characterise the lens' optical performance, a test was devised whereby light from a fibre at the focal point of the lens would be collimated. The collimated beam would then be reflected back into the lens by a plane mirror, where the lens would then re-focus the fibre image onto a detector. This test was repeated multiple times, changing the fibre size, altering the field of the lens used by rotating the mirror and varying the wavelength of the input source. It was found that the image of the $400 \mu \mathrm{m}$ fibre was re-imaged to $400 \pm 10 \mu \mathrm{m}$ and the $10 \mu \mathrm{m}$ fibre was re-imaged to $10 \pm 1.8 \mu \mathrm{m}$. Thus, proving that the optical performance of the lens alone is at the required standard.

In order to test the lens' throughput, a series of lasers were passed through the optical axis of the lens in a single pass, with the intensity of the laser measured before and after passing through the lens. The resultant attenuation for each wavelength is shown in table 3.

Table 3. Table of throughput seen for the lens on a single pass for a variety of wavelengths.

\begin{tabular}{|c|c|c|c|}
\hline Wavelength: & 532nm & 672nm & 779nm \\
\hline Throughput: & $82 \%$ & $93 \%$ & $95 \%$ \\
\hline
\end{tabular}

To summarise, lens throughput is approximately $82 \%-95 \%$ between $532 \mathrm{~nm}-779 \mathrm{~nm}$ per lens pass, this could be improved in the next iteration through the use of higher cost, higher throughput lens coatings as discussed in section 3.4.

Following confirmation that the lens performance was as required, a series of pre-prototype spectrometers were constructed using breadboarding optical mounts. These were found to be a good proof of concept but the physical size of the breadboarding mountings made setting up the spectrometer in the optimal layout close to impossible. The fibre input's fold mirror was especially difficult to mount and manipulate without beam vignette due to the compactness of that space. The entire system also had too many degrees of freedom; making alignment difficult. To combat this a custom mechanical mount was designed. 


\subsection{Mechanical mount}

In order to align the optics in the optimal way found using our Zemax model, an investigation was undertaken as to whether it would be possible to produce a mechanical mount within which we can place all of the optical components, such that optimal alignment is achievable with relative ease.

The mount was produced by taking the spectrometer alignment set-up from Zemax and exporting it as a CAD file. This CAD file was then opened in a CAD manipulation program with a derivative part created by taking the negative of the optical alignment from a 'block of CAD material'. This 'block' was then carved away, removing unnecessary parts until a basic mount shape was formed. Ideally, one could 3D print or machine this part, place the optical components in it and have the system function as designed in Zemax. Unfortunately, due to error in the manufacturing and the looseness of tolerances, we need to allow some degrees of freedom to adjust the final set-up. The required degrees of freedom were heavily scrutinised.

As an overview, the camera and fibre alignment are critical to resolution across the field, as the fibre and sensor chip must both be well aligned to the lens' focal plane. The prism, lens, input mirror and grating alignment, whilst they must not vignette, are thought to be non-critical and can be set by the model; being allowed to vary by the tolerances set on the mount manufacture.

The camera and fibre alignment must be fine tuneable, through mechanical alignment parts, but are to be designed to remain stable over long periods of time. The camera will require 3 translational d.o.f. due to uncertainty on sensor placement and 2 rotational d.o.f. to align the sensor with the focal plane. The fibre head placement is better defined and so may only require 1 translational d.o.f. perpendicular to focal plane as well as 2 rotational d.o.f.

Taking these degrees of freedom into account, a first pass CAD mount was designed, and 3D printed using PLA with a print accuracy of $100 \mu \mathrm{m}$. The resultant mount can be seen in figure 6 .

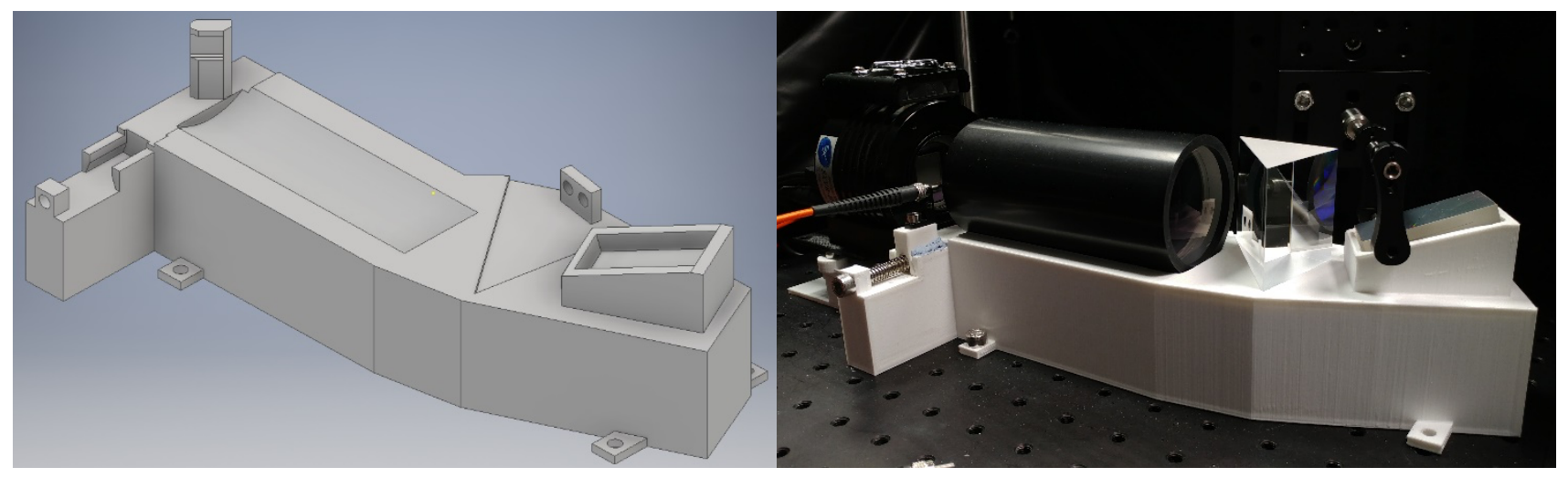

Figure 6. (Left panel) Image of CAD model mechanical mount design. (Right panel) Image of assembled 3D printed mount with spectrometer components fitted.

\section{DATA REDUCTION PIPELINE}

Whilst data reduction is achievable using IRAF, amongst other tools, and this has been tested with this spectrometer, a custom and flexible spectral extraction and data reduction pipeline has been designed for this system. Initially the pipeline 
was written with MATLAB; whilst this functions as required, it was decided that the pipeline should be able to run natively from a micro-computer such as a Raspberry Pi. This would allow the spectrometer system to be a portable standalone unit. To achieve this aim, the pipeline is in the process of being re-written in Python.

The extraction algorithm was designed to be as simplistic as possible. After dark removal, the maximum pixel values are listed in descending order for the row at the centre of the sensor (perpendicular to the diffraction orders). Moving down these values, removing the ones which are within a few pixels of each other, and hence are within the same order, the centre of each of the orders are noted until all orders are accounted for. The orders are then traced individually by looking at the neighbouring 3 pixels of the known order centre and then moving along the order to the next highest valued adjacent pixel, this process is repeated until the entire order is traced.

With the orders traced, the Flat Optimal Extraction algorithm is applied, giving the extracted orders as shown in figure 7 [19].

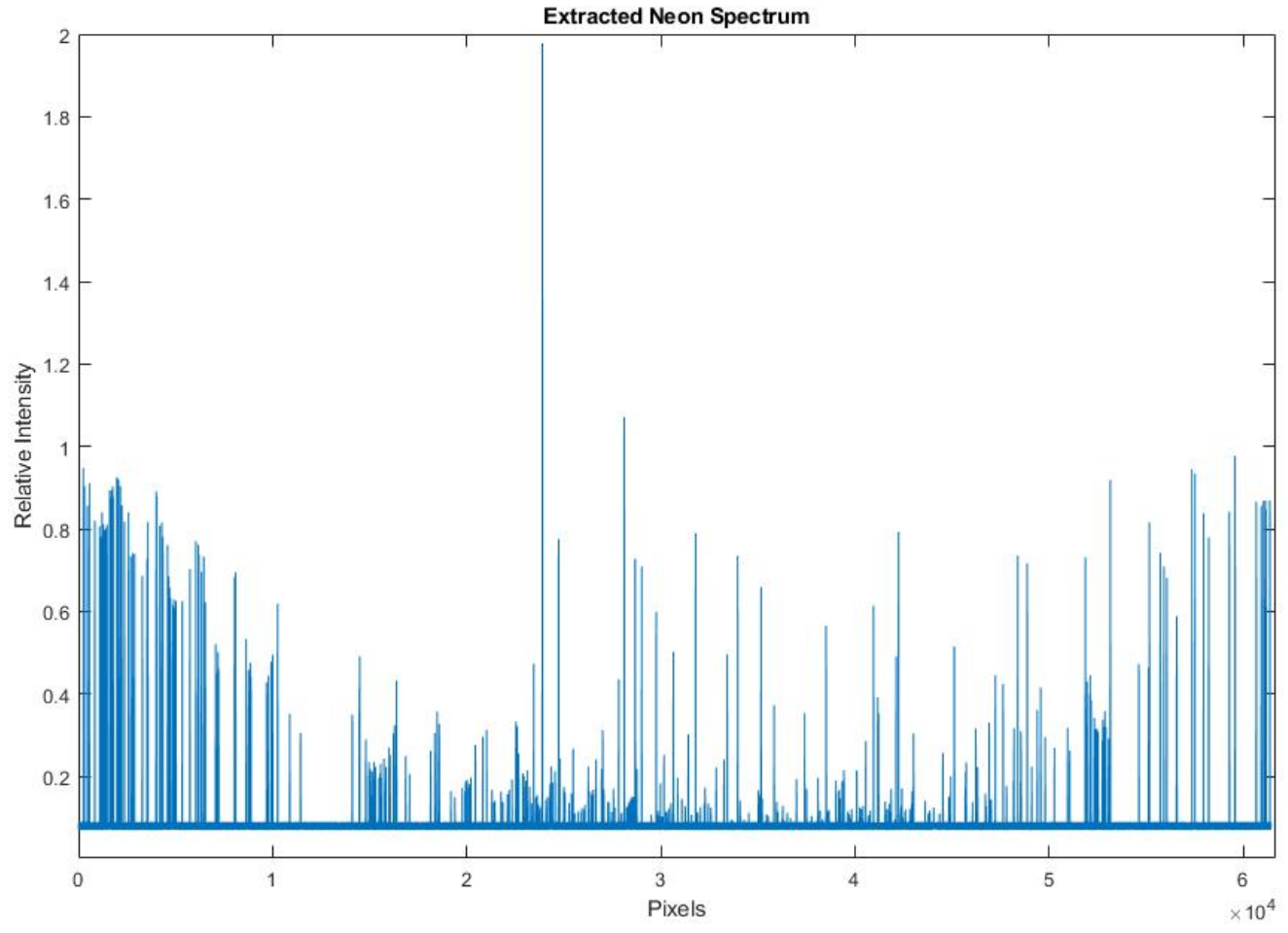

Figure 7. Extracted spectrum of Neon, with all orders plotted next to each other. Pixel number against relative intensity of the lines are plotted. The spectral lines have been masked for clarity and to remove order mismatch.

Wavelength calibration is to be achieved with a Neon lamp, but alternative calibration sources can be easily applied. The merging of orders is not necessary for our purposes. 


\section{LABORATORY TESTS}

\subsection{Mechanicals stability test using flat lamp exposures}

With the finalised iteration of the spectrometer assembled in the mechanical mounting, a brief investigation into the short and long term stability of the spectrometer was undertaken. The effects of exposure to fluctuating temperature and pressure, along with other mechanical disturbances experienced in the lab, were examined.

A flat lamp source was coupled to the fibre which feeds the spectroscope. The spectrometer was then left for an hour with a frame taken at a cadence of 6 seconds; of which, 1 second was of exposure and 5 seconds was of sensor read-out. With an initial frame chosen as a reference frame, each row of each subsequent frame was cross-correlated with the corresponding row in the reference frame in order to measure the shift from this initial frame. The peak three points of the CCF (cross-correlation function) were then used to describe a parabola with the peak of which taken as the centre of the CCF; allowing sub-pixel precision to be achieved on the shift of each row. With the centre of the CCF found for each row, the median value was taken to describe the overall shift of the image on the sensor. An example of the resultant time series can be seen in figure 8.

Time Series:

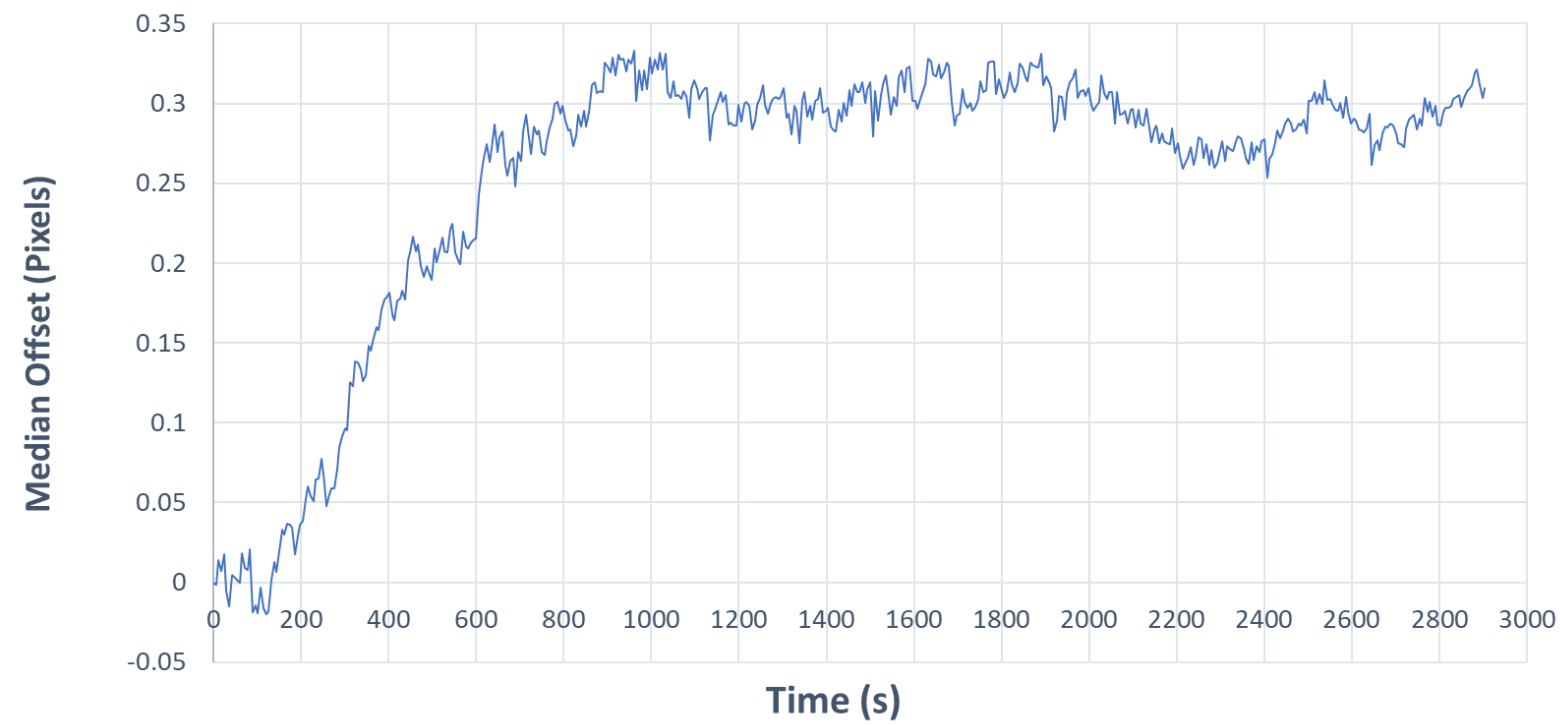

Figure 8. Graph of the time series for an example stability test. Time elapsed in seconds is plotted against median offset in pixels seen from the cross-correlation of the frame taken at a given time against the reference frame taken at $t=0$.

The test was repeated multiple times. It can be seen on figure 8 that when the spectrometer is left without interference, after approximately 15 minutes the system oscillations level out. The following analysis is undertaken on the 'level' section of the time-series. The point to point RMS was measured for this time-series to be $9.1 \pm 0.01 \times 10^{-3}$ pix. Gaussian noise was then injected into this data to represent random photon noise. The point to point RMS of this time-series with noise was found to be unchanged within the margin of error. This suggests that the stability is not photon noise dominated. 
Allan-Variance analysis of the system, computing the variance using pairs of points separated by a varying time-lag, found that stability variability is not consistent over the course of this time-series; see figure 9 . This further suggests that the stability of the system is primarily affected by either systematic error or under-sampling.

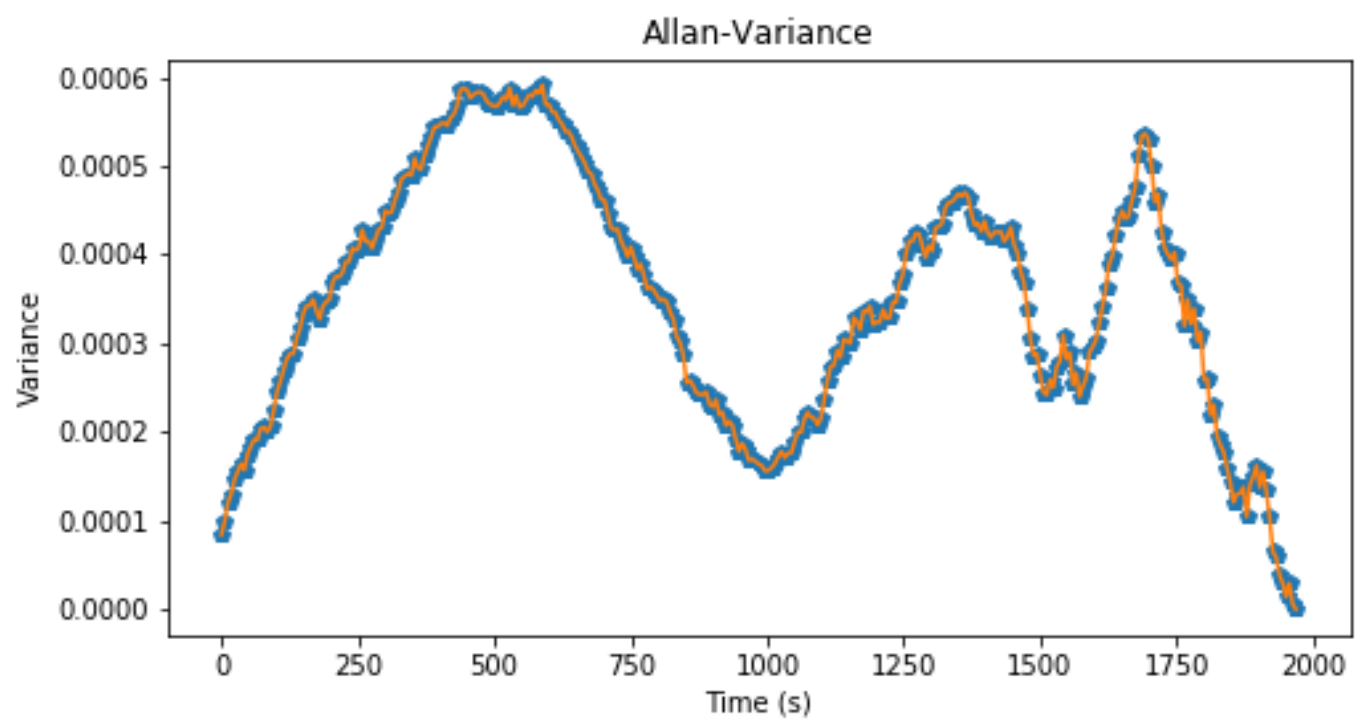

Figure 9. Graph of the Allan-Variance analysis undertake on the 'level' section of the time series seen in figure 8. Time-lag used in the variance calculation (in seconds) is plotted against variance.

Interestingly, in these tests it is seen that the system's stability has a high variance value for a time-scale of 5-10 minutes. The laboratory is located above a London Underground train-line and the mechanical disturbance of the trains passing is noticeable. This time-scale aligns well with the train timetable, so further tests in another location should be carried out to determine whether this variance is purely mechanical.

Using Doppler theorem, we see that $v / c=\delta \lambda / \lambda=1 / R$, where $c$ is the speed of light. Hence, velocity span is given by $v=c / R$. Taking, for example, a resolving power, $R$, of 60,000 we ascertain a velocity span of $v=5 \mathrm{kms}^{-1}$. With a spot size of $14 \mu \mathrm{m}$ we can estimate our resolution element to be approximately 2 pixels, therefore the velocity span per pixel is $v /$ pixel $=2.5 \mathrm{kms}^{-1}$. Our RMS point to point variability is $9.1 \pm 0.01 \times 10^{-3}$ pix, multiplying this with $v /$ pixel gives us the pixel variability in radial velocity of $r v=22.9 \mathrm{~ms}^{-1}$. As we have taken point to point values for our measurement, there is also uncertainty in the location of both points, which add in quadrature, as such a better estimate of radial velocity stability is given by dividing by a factor of $\sqrt{2}$, yielding $r v=16.2 \mathrm{~ms}^{-1}$.

Given that the system currently has no stability control system beyond the passive mechanical stability of the optical bench, and that the stability is currently dominated by systematic error, the radial velocity performance has scope to improve substantially. By reducing the point to point variability by a factor of 8 we would reach the system goal. We are confident this can be achieved with an active temperature control system, a vacuum set-up and improved mechanical isolation. 


\subsection{Blaze angle inconsistency}

It was found that the measured separation between the sodium D-lines doublet in the spectrum was lower than theoretically expected. The separation was measured on Littrow to be $666 \mu \mathrm{m}$. Using $m \delta \lambda=\varepsilon d \cos (\alpha)$, derived in section 2.1, we can see that the grating dispersion angle is given by $\varepsilon=m \delta \lambda / d \cos (\alpha)$. By rearranging the grating equation, it can be seen that $m / d=2 \sin (\alpha) / \lambda$, hence substituting this into our equation for grating dispersion, we find that $\varepsilon=2 \frac{\delta \lambda}{\lambda} \tan \alpha$. Hence, the separation seen at the focal plane for a difference in wavelength $\delta \lambda$ is given by

$$
S=\varepsilon \times f=2 f \frac{\delta \lambda}{\lambda} \tan \alpha .
$$

Using the values from the sodium doublet and the spectrometer parameters, $\delta \lambda=0.6 \mathrm{~nm}, \lambda=589 \mathrm{~nm}, f=108.24 \mathrm{~mm}$ and $\alpha=75 \mathrm{deg}$, we find that the predicted separation is $S=840 \mu \mathrm{m}$. Given this, there is a disparity between the measured and theoretical separation of the Sodium doublet. Let it be noted that even though the sodium doublet in not on Littrow for this set-up, the change in separation seen by moving up the diffraction order is a higher order effect and has minimal effect on the separation value seen here, hence it has been ignored here for brevity.

In order for the theoretical separation to match the measured value, the blaze angle would have to be set to $71 \mathrm{deg}$. To investigate whether this was the case, the grating angle was increased using a micrometer until the required separation was achieved. It was found that the intensity of the sodium doublet decreased by over a factor of 5 when at this grating angle. If this intensity reduction was caused by vignette due to the decrease grating surface projection, the intensity should only fall by a factor of 0.2 . As such, it would appear that the manufactured blaze angle of the grating varies from the quoted value and it is possible that the manufactured blaze angle has a tolerance of a few degrees. This is compounded by prior investigation which found that for the $63 \mathrm{deg}$ blaze angle Thorlabs Echelle grating, the laboratory set-up only matched the Zemax model when the model's grating angle was set to 66deg. It was also found that altering the grating in the lab system to $63 \mathrm{deg}$, incurred a substantial decrease in spectral intensity, suggesting that the blaze angle for that grating is more likely $66 \mathrm{deg}$. Further investigation and discussion with Thorlabs on this topic should be undertaken for conclusive results.

There are two take-away points from this, as it would appear that no two grating blaze angles are the same, in the next mechanical mount design, the grating angle should be made an alterable degree of freedom. Secondly, the performance of our system is currently limited by the availability and quality of the diffraction grating. We could achieve high resolution of the order $R=80,000$ with low throughput or achieve $R=60,000$ with maximal throughput. Further testing with Thorlabs gratings will be required to determine whether we can obtain one with a blaze angle of $75 \mathrm{deg}$ (or greater) in order to gain the resolving power achievable at nominal throughput.

This suggests that to further the replicability of this system or systems like this, the investment should be made into having a template grating manufactured to the required specifications. Whilst this would cost in the order of $\$ 10,000$ to procure, replica gratings could then easily be produced in the order of $\$ 100$. Along with the current lens production offering, this would lead to a low cost and easily replicable system.

\section{RESULTS}

A prototype, low-cost, high-resolution spectrometer has been produced as depicted in figure 10. The total spectrometer's footprint is $300 \mathrm{mmX} 140 \mathrm{mmX} 90 \mathrm{~mm}$, fitting well within the technical requirements. Beyond ground-based telescopes (of arbitrary size, if moderate AO is available), the instrument could also be easily implemented on space-based telescopes of arbitrary size. For example, with a minimal re-design of the mechanical mount, the system would easily fit within a $3 \mathrm{U}$ cube-sat chassis and possibly even a $2 \mathrm{U}$ chassis with a re-working of the optical design. 
The total parts cost of this prototype was less than $\$ 10,000$ but if produced as part of a larger batch, this would be reduced to below $\$ 5,000$; reaching our goal of a low-cost spectrometer.

The system has been successfully designed to achieve the goal wavelength coverage of 500nm-1000nm at close to diffraction limited performance across this range.

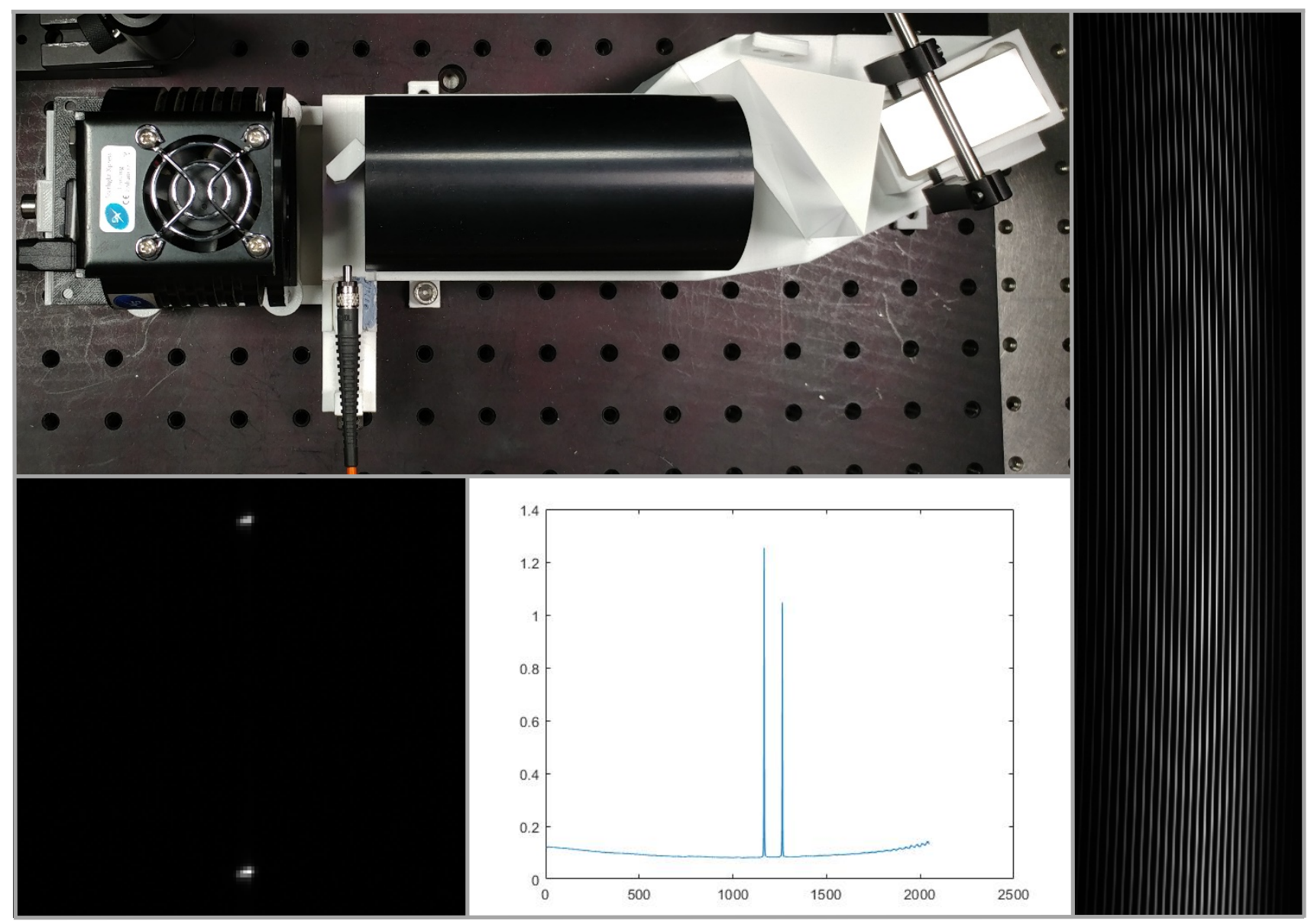

Figure 10. (Top-left panel) Image of the spectrometer assembled in the 3D printed mechanical mounting following the optical layout shown in figure 5. (Right panel) A section of the CCD image displaying the full Echelle spectra of a flat lamp. (Bottom-left panel) A section of the CCD image displaying the spectrum of the Sodium D-doublet. (Bottom-middle) The unprocessed extracted spectra of the Sodium D-doublet where pixel number (x-axis) is plotted against relative intensity (yaxis).

Mounted in the current prototype 3D-printed mechanical mount, in an on Littrow set-up, the spectrometer performs with a resolving power of $R=60,000$, surpassing the technical requirement. To go beyond this and reach the goal of $R=$ 100,000 , the current limitations have been identified as the diffraction grating and the stability/adjustability of the mount.

Analysis suggests that the grating limits performance in 2 ways: The manufactured blaze angle of the grating appears lower than expected, such that to achieve the required throughput, the spectral separation is decreased, decreasing resolution. Secondly, the grating is only $50 \mathrm{~mm}$ in height as opposed to ideal $100 \mathrm{~mm}$, causing some of the collimated beam to vignette. The vignette of the beam alone isn't a great issue but this also acts as an aperture in the dispersion direction, which limits 
the spot size to $11 \mu \mathrm{m}$ in the dispersion direction irrelevant of how good the aberration performance of the optics are. One solution to this issue is to have a template grating produced with the optimal parameters for systems like this, allowing replica gratings to be produced at a low cost. Alternatively one could apply a Virtually Imaged Phase Array (VIPA) as the high-resolution dispersing element [20]; further study into this option appears to be fruitful.

The current prototype mount was designed to minimise degrees of freedom but it is missing some necessary adjustable parameters, such as grating blaze angle. In addition to this, due to the relatively loose tolerances in the manufacturing of this mount, some alignment parts, such as the fibre mounting, whilst they should be ideally aligned as per the CAD model, are partially misaligned of the order of $0.3 \mathrm{deg}$. Future designs should either have tighter tolerances or have microadjustment for such parameters.

For the parameters which do have degrees of freedom, the translational stages function well but the rotational degrees of freedom are too coarse. A further redesign would give us the accuracy in alignment to achieve maximum performance across the CCD.

Regarding stability, measurements with precision down to a radial velocity of $16.2 \mathrm{~ms}^{-1}$ are achievable with the current set-up. This must be reduced by a factor of 4 to reach the technical requirement and a factor of 8 to reach the goal. The current system is exposed to all laboratory variations. It has no temperature or pressure control system and is under the influence of mechanical variations of the environment. As such, it would be fair to speculate that with a stability control system and a mount constructed of a more robust material (such as high-carbon steel and carbon-fibre), the stability of the system could be improved substantially. The long-term stability of the system requires further study.

The throughput of the system also requires further study. A well-educated estimate for the current system, taking the grating efficiency to be approximately $40 \%$, is of the order of $25 \%$. Efficiency of the fibre itself and CCD are not included in this value. The grating is the most lossy component of the system, as even with a blazed grating, if the grating facets are not manufactured to be sharp, a curvature on the edge of the facets can send light into other direction. As has been observed with this grating.

\section{CONCLUSION}

This prototype spectrometer has proven the concept that a compact, low-cost spectrometer can be produced in a replicable fashion. Devices such as this could be coupled to enthusiast grade telescopes across the world, opening the opportunity to be involved with high-resolution spectroscopic science to a wide audience. Alternatively, systems like this could be effectively coupled to some of the world's largest telescopes, allowing banks of these low-cost, high-resolution spectrometers to be employed in an integral field array; opening up many exciting new science cases. One such science case would include space-based high-resolution spectroscopy.

\section{REFERENCES}

[1] Faber et al., "The DEIMOS spectrograph for the Keck II Telescope: integration and testing," Proc. SPIE 4841, 1657-1669 (2003).

[2] C. Kintziger, G. Rauw, R. Desselle, P. Rochus, and Jérôme Loicq, "NGSIE: a fiber-fed near-infrared spectrograph for TIGRE telescope", Proc. SPIE 10702, 107024Y (2018). 
[3] Struve, O., "Proposal for a project of high-precision stellar radial velocity work," The Observatory, Vol. 72, p. 199-200 (1952).

[4] Nagaoka, H.; Mishima, T., "A Combination of a Concave Grating with a Lummer-Gehrcke Plate or an Echelon Grating for Examining Fine Structure of Spectral Lines," Astrophysical Journal, Vol. 57, p.92 (1923).

[5] Foust, J., "JWST suffers another launch delay, breaches cost cap," Space News, 27 June 2018, https://spacenews.com/jwst-suffers-another-launch-delay-breaches-cost-cap (17 July 2019).

[6] Snellen, I. et al, "Combining high-dispersion spectroscopy with high contrast imaging: Probing rocky planets around our nearest neighbors," Astronomy \& Astrophysics, Vol. 576, id.A59, 9 (2015).

[7] Fischer, D. A., "State of the Field: Extreme Precision Radial Velocities," The Astronomical Society of the Pacific, Vol. 128, p. 964 (2016).

[8] Dekker, H. et al., "Design, construction, and performance of UVES, the echelle spectrograph for the UT2 Kueyen Telescope at the ESO Paranal Observatory," Proc. SPIE 4008 (2000).

[9] Pepe, F. et al., "HARPS: a new high-resolution spectrograph for the search of extrasolar planets," Proc. SPIE 4008 (2000).

[10] Crane, J. D. et al., "The Carnegie Planet Finder Spectrograph," Proc. SPIE 6269, 626931 (2006).

[11] Schroeder, D. J., [Astronomical Optics Second Edition], Academic Press, San Diego, 325 (2006).

[12] Welford, W. T., [Aberrations of Optical Systems], Taylor \& Francis, New York, 30 (1986).

[13] Churchill, C., "Spectrographs," NMSU Lectures, 30 December 2009, http://astronomy.nmsu.edu/cwc/Teaching/ASTR605/Lectures/spectra.pdf (17 July 2019).

[14] Mungan, C. E., "Angle of Minimum Deviation through a Prism," USNA, 1 April 2001, https://www.usna.edu/Users/physics/mungan/ files/documents/Scholarship/MinimumDeviation.pdf (17 July 2019).

[15] Welford, W. T., [Aberrations of Optical Systems], Taylor \& Francis, New York, 237 (1986).

[16] Kingsview Optical, Optics Manufacturer, https://www.kingsviewoptical.com (17 July 2019).

[17] Schott AG, "Optical Glass Catalogue," NMSU Lectures, 1 October 2011, https://www.schott.com/d/advanced optics/094a6f8c-f648-45cd-a5fe-ca7df5474fcb/1.2/schott-optical-glasspocket-catalog-europe-october-2011-eng.pdf (17 July 2019).

[18] ATIK Cameras, “Atik 490EX Camera," 20 May 2018, https://www.atik-cameras.com/product/atik-490ex (20 May 2018).

[19] Zechmeister, M. et al., "Flat-relative optimal extraction. A quick and efficient algorithm for stabilised spectrographs," Astronomy \& Astrophysics, Vol. 561, id.A59, 8 (2014).

[20] Bourdarot, G. et al., "Experimental test of a $40 \mathrm{~cm}$-long $\mathrm{R}=100000$ spectrometer for exoplanet characterisation," Proc. SPIE 10702, 107025Y (2018). 